\title{
Using Body Mass Index to Predict Needle Length in Fluoroscopy-Guided Lumbar Punctures
}

\author{
(D)A.P. Nayate, (DI.M. Nasrallah, (D).E. Schmitt, and (D) S. Mohan
}

\begin{abstract}
BACKGROUND AND PURPOSE: Predicting the appropriate needle length to use in oblique interlaminar-approach fluoroscopyguided lumbar punctures in patients with a large body mass index is difficult. Using the wrong needle length can lead to an increased radiation dose and patient discomfort. We hypothesized that body mass index could help determine the appropriate needle length to use in patients.
\end{abstract}

MATERIALS AND METHODS: We randomly selected patients who underwent oblique interlaminar-approach fluoroscopy-guided lumbar punctures and had cross-sectional imaging of the lumbar spine within 1 year of imaging $(n=50)$. The distance from the skin to the midlumbar spinal canal (skin-canal distance) at the level of the lumbar puncture was measured by using an oblique angle of $8.6^{\circ}$, which is an average of angles most often used to perform the procedure. A formula was devised using the skin-canal distance and body mass index to predict the appropriate needle length, subsequently confirmed in 45 patients.

RESULTS: The body mass index and skin-canal distance were significantly higher $(P<.001)$ in patients who underwent fluoroscopy-guided lumbar puncture with 5 - or 7-inch needles $(n=22)$ than in patients requiring 3.5-inch needles $(n=28)$. Using linear regression, we determined the formula to predict the needle length as Skin-Canal Distance (inches) $=0.077 \times$ Body Mass Index +0.88 . We found a strong correlation $(P<.001)$ between the predicted and actual skin canal distance in 45 patients, and our formula better predicted the skin-canal distance than others.

CONCLUSIONS: We designed a formula that uses body mass index to predict the appropriate needle length in oblique interlaminarapproach fluoroscopy-guided lumbar punctures and validated it by demonstrating a strong correlation between the predicted and actual skin-canal distance.

ABBREVIATIONS: $\mathrm{BMI}=$ body mass index; $\mathrm{BMIC}=\mathrm{BMI}$ category; $\mathrm{FGLP}=$ fluoroscopy-guided lumbar puncture; $\mathrm{LP}=$ lumbar puncture; $\mathrm{OIA}=$ oblique interlaminarapproach; $\mathrm{SCD}=$ skin-canal distance

$\mathbf{T}$ he palpation of bony landmarks required to successfully perform lumbar punctures (LPs) ${ }^{1}$ can be masked in $33 \%$ of overweight patients and $68 \%$ of patients with obesity ${ }^{2}$ due to overlying soft tissue and can lead to an LP failure rate of $19 \%{ }^{3}$ without image guidance. Sonography can be helpful for guidance but can only visualize pertinent bony landmarks in patients with obesity

Received July 9, 2015; accepted after revision August 14

From the Department of Radiology, Division of Neuroradiology, Hospital of the University of Pennsylvania, Philadelphia, Pennsylvania.

Findings from this research previously presented at: Annual Meeting of the American Society of Neuroradiology and the Symposium of the ASNR Foundation, April 25-30, 2015; Chicago, Illinois.

Please address correspondence to S. Mohan, MD, Hospital of the University of Pennsylvania, Department of Radiology, Division of Neuroradiology, 3400 Spruce St, 110 Donner Building, Philadelphia, PA 19104; e-mail: suyash.mohan@uphs. upenn.edu

三 Indicates article with supplemental on-line table.

http://dx.doi.org/10.3174/ajnr.A4579
$74 \%$ of the time. ${ }^{2}$ Fluoroscopy-guided LP (FGLP) can help alleviate this issue because fluoroscopy helps the operator visualize the bony structures, irrespective of the body habitus, and, in real time, can help the operator guide the needle from the soft tissues to the spinal canal.

An oblique interlaminar approach (OIA) is a common FGLP technique, which allows the operator to maximize visualization of the interlaminar space and bypass normal and abnormal osseous obstacles, such as spondylosis. ${ }^{4} \mathrm{~A} 3.5$-inch-long (8.9-cm) LP needle is most often used to perform this procedure in adults; however with the obesity epidemic in the United States, ${ }^{5}$ the use of 5-inch-long $(12.7 \mathrm{~cm})$ and 7-inch-long $(17.8 \mathrm{~cm})$ needles is becoming increasingly common and has been estimated to be necessary in $13.8 \%$ of patients. ${ }^{6}$ Predicting the appropriate needle length to use in OIA-FGLP in patients with a larger body mass index (BMI) is difficult and primarily relies on the operator's experience. A needle that is too long may lead to an increased risk 
of piercing the anterior epidural space, which can cause a traumatic tap, ${ }^{7}$ while a needle that is too short may not reach the spinal canal, requiring the insertion of a longer needle and potentially increasing patient discomfort and fluoroscopy time/radiation dose. An increased radiation dose is a particular concern in patients with obesity because increasing BMI is directly related to higher fluoroscopy times in patients undergoing FGLP. ${ }^{8}$ In 2011, The Joint Commission expressed concern about the exposure of the American population to medical imaging ionizing radiation and recommended reviews of practices to reduce radiation exposure to as low as reasonably achievable without compromising patient care. ${ }^{9}$

Formulas to determine proper needle length in non-fluoroscopy-guided interspinous lumbar punctures have been investigated $^{10-13}$; however, there is no established method to determine the appropriate needle length for OIA-FGLPs. The purpose of this study is to determine whether BMI could help predict the appropriate needle length in OIA-FGLPs.

\section{MATERIALS AND METHODS}

This study was approved by the local institutional review board.

\section{Procedure Technique}

Following informed consent, all patients underwent FGLP in the prone position by using a standard biplanar fluoroscopy machine in a neurointerventional suite. The procedures were performed by using techniques as dictated by the American College of Radiology-American Society of NeuroradiologySociety for Pediatric Radiology practice parameters. ${ }^{14}$ LPs were performed by first-year neuroradiology fellows (fellows had little or no prior experience in performing FGLPs before the fellowship) under the supervision of attending neuroradiologists (experience in FGLPs ranged from 1 to $20+$ years).

Under fluoroscopy, the lumbar levels were visualized and the $\mathrm{x}$-ray tube was maneuvered to the right or left oblique orientation to optimize the view of the interlaminar space as determined by the operator. Using a 3.5-, 5-, or 7-inch beveled tip 22-ga needle, we accessed the lumbar spinal canal mostly at the L2-L3 or L3-L4 level as instructed by the American College of Radiology-American Society of Neuroradiology-Society for Pediatric Radiology practice parameters. ${ }^{14}$ Briefly, these levels are favored because the distance of the skin to the subarachnoid space is shorter ${ }^{6}$ and the thecal sac cross-sectional area is larger compared with L4-L5 or L5-S1, ${ }^{15}$ while there is a higher rate of traumatic puncture ${ }^{7}$ from LPs at L4-L5 and a higher incidence of degenerative changes and spinal canal stenosis at L4-L5 and L5-S1. ${ }^{16}$ At our institution, LPs are rarely performed at L4-L5 or L5-S1. L1-L2 was never accessed due to the risk of injuring the spinal cord.

The choice of the needle length was determined by the operator on the basis of the patient's body habitus. Patient BMIs were not routinely checked before the LP. This check is not a standard practice at our institution or explicitly stated by the American College of Radiology-American Society of NeuroradiologySociety for Pediatric Radiology guidelines. ${ }^{14}$

The needle was advanced through the skin into the lumbar spine with intermittent pulsed fluoroscopy. Access into the thecal sac was confirmed on egress of CSF after the removal of the stylet.

\section{Determination of Tube Angulation}

The oblique angle of the $\mathrm{x}$-ray tube used for FGLP at our institution from September 30 to November 4, 2014, was recorded in a consecutive subset of patients ( $n=30$; age range, 19-69 years of age; 14 females and 16 males). In 5/30 patients, a single operator used a craniocaudal angle (range, $7^{\circ}-27^{\circ}$ ) in addition to the lateral obliquity. The lumbar punctures were performed at L2-L3 in 14 patients, at L3-L4 in 15 patients, and at L4-L5 in 1 patient. The average oblique angle used to perform the lumbar punctures was $8.6^{\circ} \pm 5.2^{\circ}$.

\section{Patients with Imaging of the Lumbar Spine (Derivation Sample)}

All patients who had FGLP at our hospital during 11 consecutive months from August 2013 to June 2014 were retrospectively reviewed. FGLP was performed on 322 patients (3.5-inch needle, $n=279$; 5 -inch needle, $n=36$; and 7-inch needle, $n=$ 7). In all patients, age, sex, indication for lumbar puncture, anatomic level of lumbar puncture, and total fluoroscopy time were recorded.

We randomly selected 50/279 patients from the 3.5-inch needle set and included all the patients in the 5- $(n=36)$ and 7-inch $(n=7)$ needle sets for further analysis (total, $n=93)$. All 50 patients had successful FGLP without adverse events. Our institutional PACS was queried to identify from these 93 patients those who had cross-sectional imaging of the abdomen or lumbar spine (CT, MR imaging, PET/CT covering the abdomen, or CT or MR imaging of the lumbar spine) performed within 1 year of the LP. Inclusion criteria for cross-sectional imaging were supine acquisition and inclusion of the flank skin and soft tissue, lumbar spinal canal, and lumbar vertebral bodies in the FOV. Fifty subjects of the 93 in the study met these criteria: $28 / 50$ patients in the 3.5 -inch group, $19 / 36$ patients in the 5 -inch group, and $3 / 7$ patients in the 7-inch group. Additionally, the patient's BMI obtained closest to the day of the FGLP and lumbar or abdominal imaging and days between imaging and LP were recorded. BMI was categorized (BMI category $[\mathrm{BMIC}])$ as underweight $(\mathrm{BMI}<18.5)$, normal $(\mathrm{BMI}=18.5-24.9)$, overweight $(\mathrm{BMI}=25-29.9)$, obese $(\mathrm{BMI}=$ $30-39.9$ ), and extremely obese (BMI $\geq 40$ ), according to the obesity guidelines of the National Heart, Lung, and Blood Institute. ${ }^{17}$

\section{Measurement of Skin-to-Mid-Spinal Canal in the Patients with Lumbar Spine Imaging}

We measured the distance from the skin to the midpoint of the spinal canal (skin-canal distance [SCD]) at the level where the lumbar puncture was performed in our cohort of 50 patients by using the standard measurement tools available on our PACS. To account for potential variations in needle approach, we measured the SCD at several angles, including parallel to and intersecting the spinous process (eg, standard bedside interspinous approach lumbar puncture angle, $0^{\circ}$ ) at the empirically determined mean oblique angle $\left(8.6^{\circ}\right)$ and at angles $1 \mathrm{SD}$ from the mean angle $\left(3.4^{\circ}\right.$ and $\left.13.8^{\circ}\right)$. We excluded the craniocaudal 


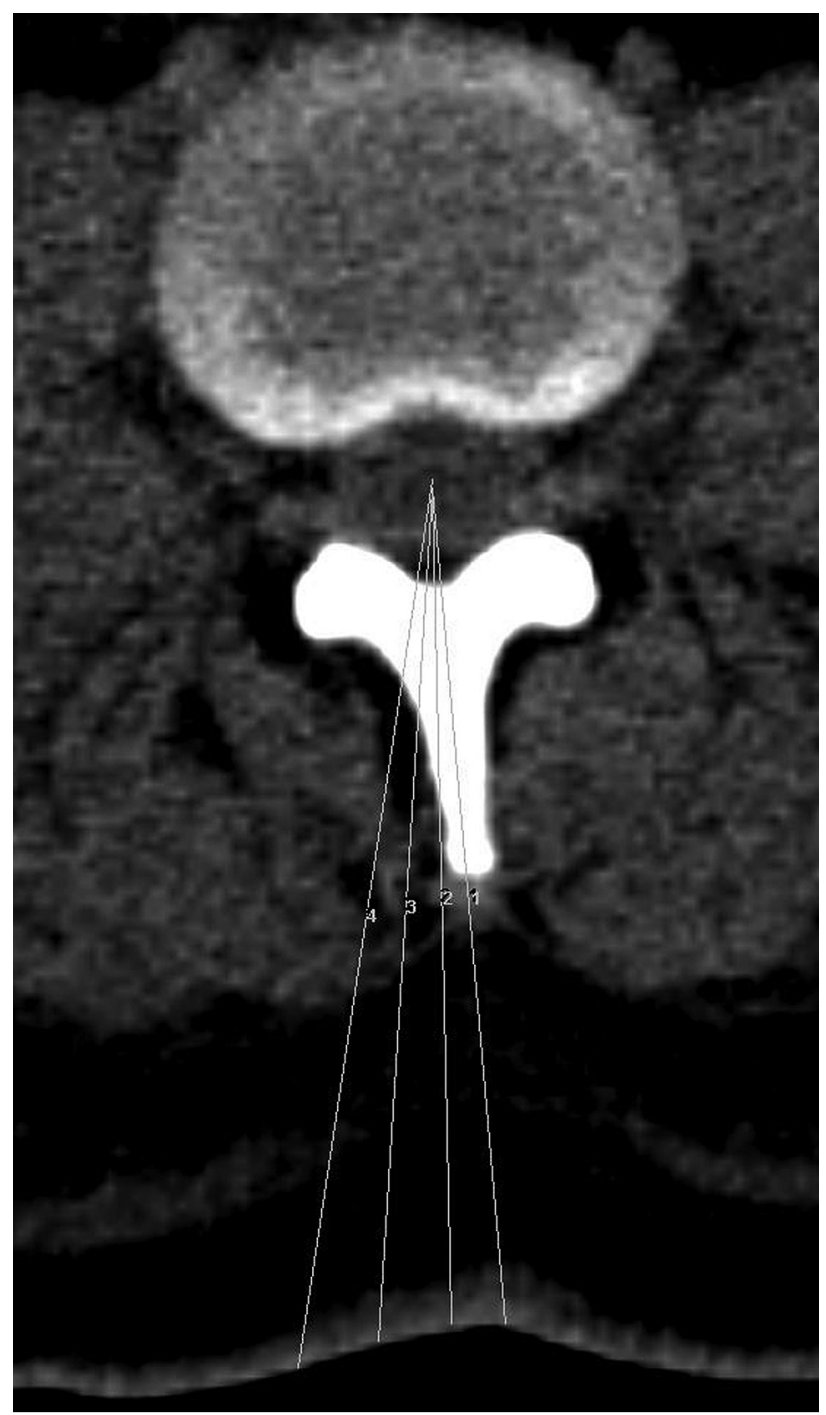

FIG 1. Angles used to measure skin to midlumbar spinal canal. Labels: $1=0^{\circ}, 2=3.4^{\circ}, 3=8.6^{\circ}, 4=13.8^{\circ}$.

angles to simplify the measurements. Figure 1 summarizes the measurement process.

\section{Confirmation Sample}

For confirmation, in a sample of 45 patients ( $n=16$ at L2-L3; $n=$ 21 at L3-L4; and $n=8$ at L4-L5) who underwent OIA-FGLP from December 2014 to May 2015, the SCD was physically measured. Specifically, the tip of the needle was confirmed to be in the center of the spinal canal with a single lateral view. After the completion of the FGLP, the LP needle was marked at the skin and removed, and the distance from the skin surface to the tip of the needle was measured. BMIs obtained closest to the day of FGLP and other demographic measures were acquired from the electronic medical record as described above. To minimize selection bias, we required no inclusion criteria for our confirmation sample, including prior cross-sectional imaging. One patient was included in the confirmation sample and in the derivation sample; the remaining 44 patients were separate from the derivation sample.

\section{Statistical Analysis}

Demographic information was summarized with means for continuous variables and proportions for discrete variables. $T$ tests were performed to compare group differences in BMI and fluoroscopy time. To estimate the effect of potentially variant needle angles on the reliability of CT- and MR imaging-derived SCD measurements, we calculated the intraclass correlation coefficient among measurements at different angles $\left(0^{\circ}, 3.4^{\circ}, 8.6^{\circ}, 13.8^{\circ}\right)$. Following graphic display of the data and assessment for normality, linear regression was used to explore the relationship between BMI and SCD. The $\beta$ weights from the best-fit model were used to construct a formula for predicting needle length from BMI alone. On the basis of this formula, SCD was estimated for our confirmation sample on the basis of BMI and was compared with the actual measured SCD length via linear regression. Repeated-measures ANOVA was used to compare the deviation between the predicted and actual SCD between our formula and others. Multiple regression was used to examine the relationship between fluoroscopy time and needle length after accounting for BMI. For hypothesis tests, a 2 -tailed $\alpha$ of .05 was defined as the threshold for statistical significance.

\section{RESULTS}

\section{Patients with Imaging of the Lumbar Spine (Derivation Sample)}

In our sample of subjects with both LP and cross-sectional imaging data, there were 18 women and 32 men with a mean age of $49.1 \pm 17.2$ years. Twenty-eight patients had LP at L2-L3; 17 patients, at L3-L4; 4 patients, at L4-L5; and 1 patient, at L5-S1. The indications for the LP were the following: 23 patients, intrathecal chemotherapy; 15 patients, CSF tumor cell detection; 1 patient, evaluation for neurosarcoidosis; 6 patients, detection of an infectious/inflammatory process in the CSF; 3 patients, concern for pseudotumor cerebri; 1 patient, indeterminate cervical spinal cord lesion; and 1 patient, concern for subarachnoid hemorrhage.

Patients had cross-sectional imaging of the abdomen or lumbar spine an average of 90 days (range, 1-353 days) before or after the LP. The cross-sectional imaging consisted of the following: 34 patients with CT of the abdomen, 9 patients with MR imaging of the lumbar spine, 5 patients with PET/CT, 1 patient with MR imaging of the abdomen, and 1 patient with CT of the lumbar spine.

In our sample of 50 patients, based on BMI, 2 patients (2\%) were characterized as underweight; 11 patients (22\%), as having normal weight; 11 patients (22\%), as overweight; 19 patients (38\%), as obese; and 7 patients (14\%), as extremely obese. Patients, on average, had a BMI obtained within 12 days of their FGLP and within 17 days of their cross-sectional lumbar or abdominal imaging. These $2 \mathrm{BMI}$ values were not significantly different (average difference in BMI, 1.4; range, $0-6.4 ; P=$ .78 ), nor were the BMI of men (average BMI $=30.7 \pm 11$ ) compared to women (average BMI $=33.34 \pm 9.5$ ), $P=.38$.

The BMIs of the patients who underwent FGLP with a 5- or 7 -inch needle ( $n=22$; average, $40.1 \pm 9.8$; BMIC, extremely obese; 11 women and 11 men) were significantly higher $(P<$ .001) than the BMIs of patients who underwent FGLP with a 


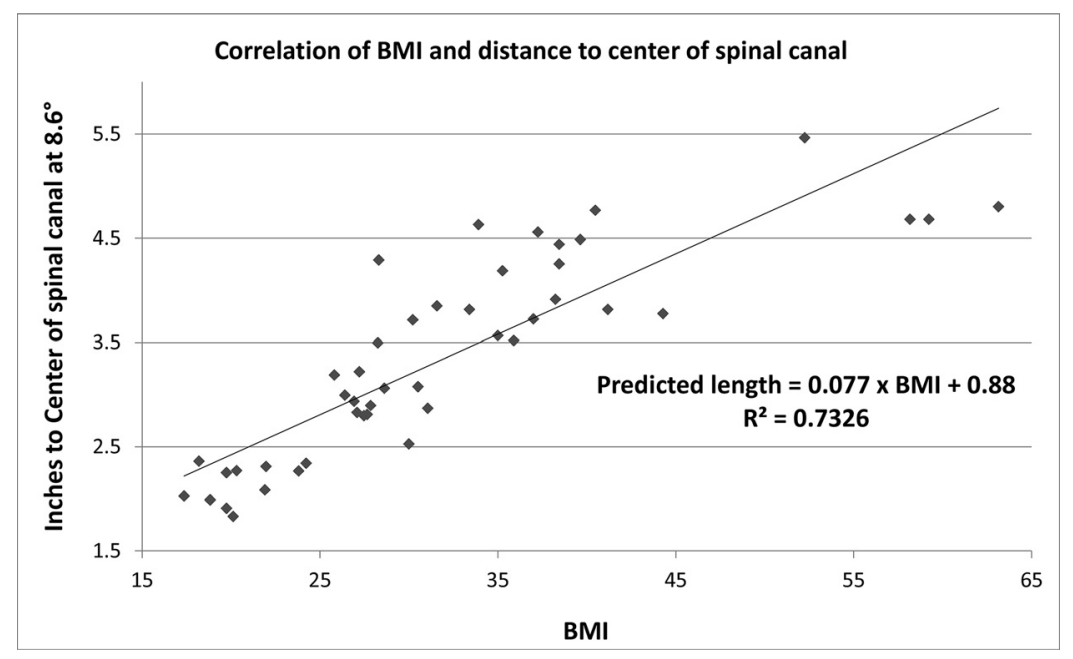

FIG 2. Correlation of 50 patient BMls and the distance to the center of the spinal canal on cross-sectional imaging.

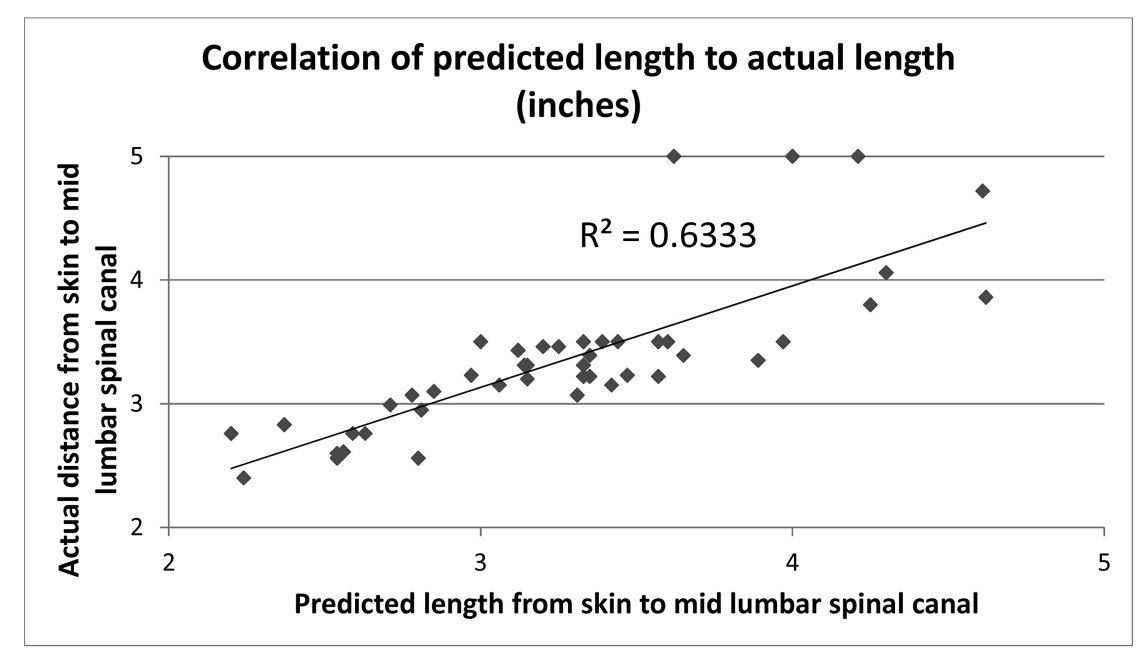

FIG 3. Correlation between actual and predicted SCDs.

3.5-inch needle ( $n=28$; average, $25 \pm 4.7$; BMIC, normal-overweight; 7 women and 21 men). The fluoroscopy time in patients who underwent FGLP with a 5- or 7-inch needle (average, $1.32 \pm$ 0.89 minutes $)$ was significantly longer $(P=.004)$ than that in patients who underwent FGLP with a 3.5-inch needle (average, $0.65 \pm 0.57$ minutes) and also in the entire cohort of patients (3.5-inch needle, $n=279 ; 5$ - and 7-inch needle, $n=43$ ) who underwent FGLP $(P=.004)$. Multiple regression confirmed that needle length was an independent predictor of fluoroscopy time, even after accounting for BMI $(P=.003)$.

\section{Measurement of the Skin-to-Mid-Spinal Canal in Patients with Lumbar Spine Imaging}

SCD estimates were highly reliable between the measured-approach angles (intraclass correlation coefficient $=0.99$ ). The distance measured on the cross-sectional imaging in the cohort of 50 patients at the mean FGLP right oblique angle of $8.6^{\circ}$ was significantly higher $(P<.001)$ in patients who underwent FGLP with a 5 - or 7-inch needle (average, $4.1 \pm 0.6$ inches) compared with patients who underwent FGLP with a 3.5 -inch needle (mean, $2.7 \pm 0.6$ inches). Similar results were seen at other angle mea- surements (all $P<.001$ ). The data obtained from the cross-sectional imaging were plotted against the BMI, and with linear fit, we determined the following formula at an oblique angle of $8.6^{\circ}$ : Skin to Center of Spinal Canal (inches) = $0.077 \times \mathrm{BMI}+0.88$ (Fig 2).

\section{Confirmation Sample}

There was a strong correlation between predicted and actual SCD in our confirmation sample $\left(r=0.80, R^{2}=0.63, P<\right.$ $.001)$, with a difference of $8.0 \% \pm 6.2 \%$ (Fig 3). There was no significant difference $(P=.76)$ between the predicted and measured lengths if the procedure was performed at L2-L3 ( $n=16$; average, $8.4 \% \pm 5.5 \%)$, at L3-L4 $(n=21$; average, $8.3 \% \pm 7.4 \%$ ), or at L4-L5 $(n=8$; average, $6.5 \% \pm 4.0 \%)$. The average SCD was not significantly different between the predicted $(3.27 \pm 0.60$ inches) and measured (3.36 \pm 0.61 inches) length difference, 0.09 inches; $P=.15$. The average difference between the predicted and measured SCD length for women was $11.5 \% \pm 6 \%(n=15)$ and was significantly higher $(P=.001)$ than that for men $(6.23 \% \pm 5.4 \% ; n=$ 30 ). Our formula more accurately predicted the needle length from the skin to the spinal canal than other published formulas, which were determined for ISLPs (On-line Table). Figure 4 compares the accuracy of existing formulas in predicting the actual SCD in our confirmation sample. The current model was significantly more accurate $(P<.0001)$ compared with other formulas.

In the confirmation sample of 15 women and 30 men, the average age was $47.1 \pm 16.9$ years and the average BMI was $31.1 \pm$ 7.7 (average BMI in men, $30.6 \pm 6.7$; BMIC, overweight; average $\mathrm{BMI}$ in women, $33.0 \pm 10.1$; BMIC, overweight). On the basis of the BMI, 2 patients (4\%) were classified as underweight; 8 patients, as having normal weight (18\%); 10 patients, as being overweight (25\%); 18 patients, as being obese (40\%); and 7 patients (16\%), as being extremely obese. These values were similar to those seen in our cohort of 50 patients from which we determined the formula. The average oblique angle used was $8.5^{\circ} \pm 4.8^{\circ}(n=$ 42; for 3 patients, the angle was not recorded), similar to the $8.6^{\circ}$ angle determined from the angle-determination cohort of 30 patients.

In 36/45 patients, a 3.5-inch needle was used; in 8/45 patients, a 5 -inch needle was used; and in 1 patient, a 7 -inch needle was used. In 1 of the patients requiring a 5 -inch needle, originally a 3.5-inch needle was used; however, LP was unsuccessful and CSF was obtained after the use of a 5-inch needle. Our formula pre- 
Accuracy of BMI in predicting Source to Spinal Canal Distance

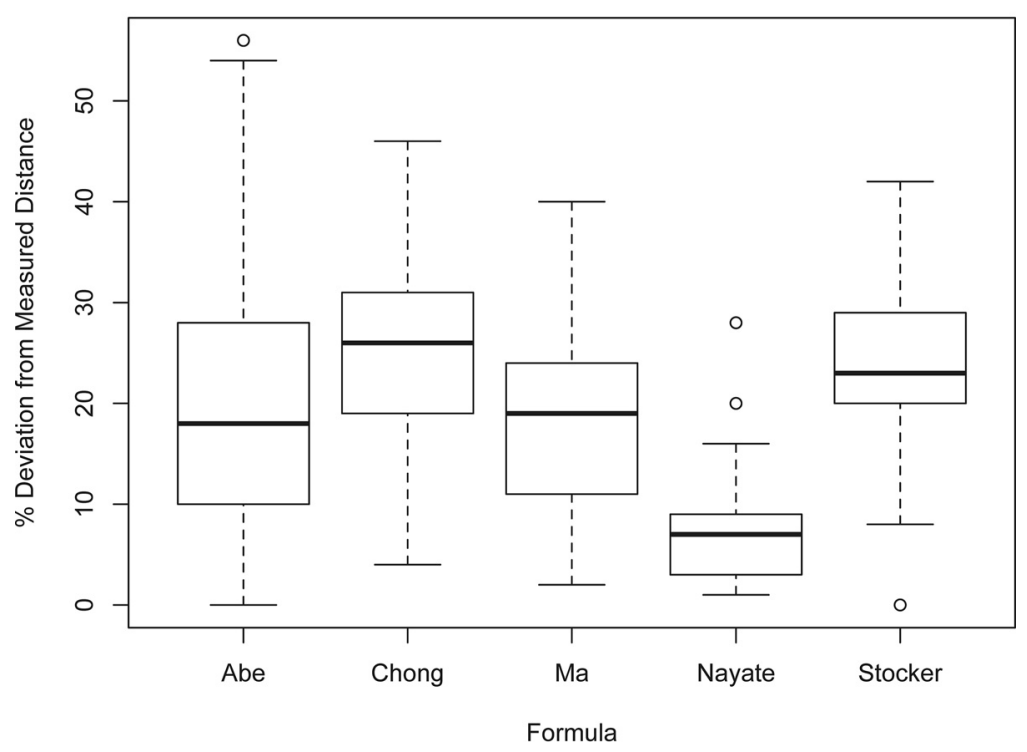

FIG 4. Boxplots demonstrating the accuracy in predicted $S C D$ as a function of the formula. The predicted SCD from our method was closer to the actual SCD in our confirmation sample compared with the other formulas $(P<.0001$ for all pair-wise comparisons, repeated measures ANOVA, Bonferroni correction).

Table 1: Accuracy of formula to predict the appropriate needle length to use in the confirmation sample ${ }^{a}$

\begin{tabular}{lcc}
\hline Actual SCD & Correct & Incorrect \\
\hline$<3.5$ inches & $28 / 31(90 \%)^{\mathrm{b}}$ & $3 / 31(10 \%)^{\mathrm{c}}$ \\
$>3.5$ inches & $7 / 7(100 \%)^{\mathrm{c}}$ & $0(0 \%)$ \\
Total & $35 / 38(92 \%)$ & $3 / 38(8 \%)$ \\
\hline
\end{tabular}

${ }^{a}$ Accuracy of formula needle-length prediction, 3.5 or 5 inches. In $7 / 45$ patients, the actual SCD was 3.5 inches; our formula predicted a 5 -inch needle in $3 / 7$ (43\%) patients and a 3.5-inch needle in $4 / 7$ (57\%) patients.

b Predicted 3.5-inch needle.

c Predicted 5-inch needle.

Table 2: Accuracy of formula versus neuroradiologists at our institution (fellow or attending, $n=19$ ) in selecting the appropriate needle length ${ }^{\mathrm{a}}$

\begin{tabular}{lcc}
\hline Actual SCD & Formula & Neuroradiologists \\
\hline$<3.5$ inches & $28 / 31(90 \%)^{\mathrm{b}}$ & $30 / 31(97 \%)^{\mathrm{b}}$ \\
$>3.5$ inches & $7 / 7(100 \%)^{\mathrm{c}}$ & $5 / 7(71 \%)^{\mathrm{d}}$ \\
Total & $35 / 38(92 \%)$ & $35 / 38(92 \%)$ \\
\hline
\end{tabular}

${ }^{a} \ln 7 / 45$ patients, the actual SCD is 3.5 inches. Our formula predicted a 5 -inch needle in $3 / 7(43 \%)$ patients and a 3.5 -inch needle in $4 / 7$ (57\%) patients, and the neuroradiologists selected a 3.5 -inch needle in 6/7 (86\%) patients and a 5-inch needle in 1/7 (14\%) patients.

${ }^{\mathrm{b}}$ Predicted 3.5-inch needle.

c Predicted 5-inch needle.

${ }^{\mathrm{d}}$ A 3.5-inch needle was unsuccessfully used in 1 patient, and a 7-inch needle, in another patient by 2 different neuroradiologists.

dicted that a 5 -inch needle would be required in this patient. In the patient in whom a 7 -inch needle was used, initially LP was unsuccessful with a 5-inch needle inserted at the L4-L5 level. Fluoroscopy showed the needle tip in center of the spinal canal, but there was no egress of CSF. LP with a 7-inch needle was successful at the L3-L4 level. The skin-to-mid-spinal canal measurement based on the LP needle was 4.72 inches, confirming that a 5-inch needle could also have been used at L3-L4; our formula predicted the SCD to be 4.5 inches. Similarly, in 1 patient, a 5 -inch needle was used at L3-L4; however, our formula predicted that a 3.5-inch could have been used, and our prediction was confirmed with measurements on the LP needle (predicted, 3.06 inches; actual, 3.15 inches) (Online Table). Table 1 shows the accuracy of our formula in needle-length prediction, and Table 2 shows a comparison between the needle-length predictions by the formula and neuroradiologists' at our institution.

\section{DISCUSSION}

Predicting lumbar puncture needle length in overweight patients and those with obesity is usually based on the operator's experience and can have a failure rate of $19 \%{ }^{3}$ without image guidance. Many of these patients with prior unsuccessful lumbar punctures are referred to the neuroradiology department for image-guided lumbar punctures under fluoroscopy. There are no established methods to help determine the appropriate needle length to use in OIA-FGLPs, and this determination primarily relies on the operator's experience.

We derived a formula to predict the appropriate needle length to use in OIA-FGLP by using measurements from the skin to the midlumbar spinal canal on lumbar cross-sectional imaging, patients' BMIs, and oblique angles most commonly used to perform the procedure. The measurements we obtained from 4 angles $\left(0^{\circ}\right.$, $8.6^{\circ}, 3.4^{\circ}$, and $\left.13.8^{\circ}\right)$ were very similar, suggesting that our formula can be used on a wide range of OIA angles and with the interspinous approach, which is much more commonly used without fluoroscopic guidance.

We validated our formula by measuring the distance from the skin surface to the needle tip in patients who underwent FGLP and found it highly predictive of actual SCD. The distance our formula predicted was, on average, 0.28 inches (8.0\%) different, not statistically significantly different from that measured $(P=$ .51). Our formula overpredicted the needle length in $13 / 45$ (29\%) patients, underpredicted it in 22/45 (49\%) patients, and precisely predicted (difference of $<0.1$ inch) it in $10 / 45(22 \%)$ patients. The underpredictions never resulted in a needle too short to reach the spinal canal, while the overpredictions would have resulted in using a longer needle than needed in $3 / 45(7 \%)$ patients (5 inch instead of 3.5 inch). In $7 / 45$ (16\%) patients, the SCD was 3.5 inches, so a 3.5- or 5-inch needle could be appropriately used.

The needle-length prediction from our formula would have guided the operator to use a 5-inch needle in a case that was unsuccessful with the use of a 3.5-inch needle and would have guided the operator to use a 5 -inch needle instead of a 7 -inch needle and a 3.5-inch instead of a 5-inch needle in 2 patients, appropriate changes as confirmed on measurements performed on the LP needles. Our formula correctly predicted the appropriate needle length to use in $92 \%$ of patients ( 3.5 inch, $n=28 / 31 ; 5$ inch, $n=7 / 7$ ) (Table 1 ) and more accurately predicted the 
appropriate use of a 5-inch needle than the subjective assessment of 2 neuroradiologists (7/7 patients versus 5/7 patients, Table 2). Neuroradiologists more accurately predicted the use of a 3.5-inch needle than the formula, but overall (the 3.5- and 5-inch-needle groups included), the formula and neuroradiologists were equal in predicting the correct needle length $(35 / 38,92 \%)$. The high accuracy of subjective needle-length prediction by neuroradiologists at our institution may be related to the extensive experience in performing FGLP, because approximately 400+ FGLPs are performed at our institution yearly. Our formula may be particularly helpful to health care practitioners who perform FGLPs less frequently.

Our formula more accurately predicted the needle length in male patients than in female patients $(6.23 \%$ versus $11.5 \%$, respectively; $P=.001$ ) and is probably due to sex differences in body fat distribution, with women having a more variable fat distribution with higher subcutaneous fat in the lower back and gluteal region, while men have more central adiposity. ${ }^{18,19}$ However, less accuracy in female patients did not translate to errors in needle selection as our formula better predicted the correct needle length in women than in men. Our formula would have predicted the correct needle length in 14/15 (93\%) female patients and selection of a needle that was too long ( 5 versus 3.5 inch) in 1 patient; while in men, our formula predicted the correct needle in 25/30 (83\%) patients and selection of a needle that was too long ( 5 versus 3.5 inch) in 5 male patients.

There have been studies that have derived formulas to estimate SCD for interspinous approach LPs, including the formulas by Abe et al, ${ }^{10} \mathrm{Ma}$ et al, ${ }^{11}$ Stoker and Bonsu, ${ }^{12}$, and Chong et al, ${ }^{13}$ which are often cited in the literature. The SCD that all 4 formulas predicted were significantly different from the actual SCD (all, $P<.001)$ measured in our cohort of 45 patients. The predicted SCD from our formula was $8 \%$ different from the actual one, while the predicted lengths from the formulas of Abe et al, Ma et al, Stoker and Bonsu, and Chong et al were 20\%, 18\%, 24\%, and $25 \%$ different from the actual SCD, respectively (Fig 4). The use of the formulas of Ma et al, Stocker and Bonsu, and Chong et al in our cohort resulted in average predicted lengths of $0.56,0.83$, and 0.85 inches less than the actual distance, respectively; and using their formulas would have resulted in selection of a needle that was too short (3.5 versus 5 inches) in $2 \%(1 / 45)$ of patients with the formula of Ma et al, 13\% (6/45) of patients with the formula of Stoker and Bonsu, and 11\% (5/45) of patients with the formula of Chong et al. The formula of Abe et al predicted an average length of 0.62 inches higher than the actual distance. Most of the differences $(41 / 45)$ were overpredictions and would have led to an unnecessary use of a longer needle length in 64\% (29/45) of patients. The unnecessary use of a longer needle can be problematic because longer needles are independent predictors of longer fluoroscopic time ( $P=.003$, in our cohort of 7 neuroradiology fellows), even after accounting for BMI, and there may be an increased risk of piercing the anterior epidural space, which can lead to a traumatic tap. ${ }^{7}$ Furthermore, anecdotally, a longer needle is more difficult to guide from the skin into the spinal canal, particularly for less experienced operators; and there is often slower egress of CSF through longer needles, potentially causing a longer procedural time.
We believe our formula more accurately predicted the SCD length than the formula of Abe et $\mathrm{al},{ }^{10}$ because the $\mathrm{BMI}$ in the cohort of 50 patients from which we derived our formula is more reflective of the adult US population. The patient population in the Abe et al study had a BMI of 22.6 (BMIC, normal) and included children and adults, while the BMI in our cohort of adult patients was 31.7 (BMIC, obese), which is more similar to the average BMI of 28.4 (BMIC, overweight) seen in the adult US population. ${ }^{20}$ We believe our formula more accurately predicted the needle length than the formula of $\mathrm{Ma}$ et $\mathrm{al}^{11}$ because in our cohort of 50 patients, the SD of weight (kilograms) of the patients was higher (24.2 versus $12.7 \mathrm{Kg}$ ). A formula derived from a wider range of patient weights suggests that the formula is potentially more applicable to a larger subset of the general population. We speculate that our formula more accurately predicted the needle length than the formulas of Stocker and Bonsu ${ }^{12}$ or Chong et $\mathrm{al}^{13}$ because children, who constituted their patient population, have shorter skin-to-mid-spinal canal measurements compared with adults and typically require 1.5-, 2.5-, or 3.5-inch needles.

Needle-length predictions from our formula are validated for FGLPs at L2-L3 and L3-L4 because 90\% of the patients from whom we devised our formula underwent LPs at these levels. This finding is consistent with the practices of our institution and most other neuroradiology practices, where most of the FGLPs are performed at these 2 levels. ${ }^{20}$ LPs are usually avoided at L4-L5 and L5-S1 due to the higher incidence of degenerative changes and spinal canal stenosis ${ }^{16}$ and the smaller cross-sectional area of thecal sac compared with L2-L3 and L3-L4 ${ }^{15}$ which could cause a lower success rate. In addition, lumbar punctures at L4-L5 are associated with twice the risk of traumatic lumbar puncture compared with L2-L3 and L3-L4, ${ }^{7}$ which could lead to complications and confound results, especially in patients with concern for subarachnoid hemorrhage. In the limited patients in whom we performed FGLPs at L4-L5, our formula was accurate in predicting the needle length in $8 / 8$ patients, with a $6.5 \%$ difference between the predicted and actual length. However, the average BMI in these patients was $25.6 \pm 5.2$ (BMIC, normal-overweight), which was lower than the average BMI in our cohort of patients who underwent LPs at L2-L3 (average BMI, $31.5 \pm 8.4$; BMIC, obese; $n=16$ ) and L3-L4 (average BMI, $32.6 \pm 7.5$; BMIC, obese; $n=$ 21) because operators favored performing FGLPs at L2-L3 and L3-L4 in patients with large BMIs because the distance from the skin to the subarachnoid space is shorter at L2-L3 and L3-L4 compared with L4-L5. ${ }^{6}$

Our study has limitations. First, our sample size $(n=50)$ from which we derived our formula was small; however, our patient population is reflective of the US population because the BMI of 31.7 (BMIC, obese) in our cohort is similar to the average BMI of 28.4 (BMIC, overweight) in the adult US population..$^{21}$ Second, our findings are based on the FGLPs performed at a single institution; though with a total of 14 neuroradiology fellows performing the FGLP under the supervision of 15 attending neuroradiologists, there was a range of operator skills. Third, our formula is designed to predict the appropriate needle length to use at the L2-L3 and L3-L4 levels and might not be accurate at L4-L5 or L5-S1, especially in patients with obesity $(\mathrm{BMI}>30)$. Fourth, on cross-sectional imaging, we measured the distance from the skin 
to the midspinal canal with the patient in a supine position, which can vary slightly (approximately 0.1 inch) from that determined from measurement in the prone position used for FGLP. ${ }^{22}$ Fifth, lumbar puncture needles can bend while traversing from the skin to the spinal canal and could potentially affect the measured distance on the needle used as the reference standard. Sixth, the accuracy of prediction of SCD diminishes in patients with extreme obesity.

\section{CONCLUSIONS}

We derived a formula $(0.077 \times \mathrm{BMI}+0.88)$ to predict the distance from the skin to the center of the spinal canal to determine the needle length (inches) to use in OIA-FGLPs in adults. We validated our formula by demonstrating only an $8 \%$ difference between the needle-length predictions and the actual distance from lumbar puncture needles used in patients. Our formula better predicted the SCD than other published formulas and can help guide operators in selecting the appropriate needle length for FGLPs.

Disclosures: James E. Schmitt-UNRELATED: Grants/Grants Pending: Radiological Society of North America (current) and National Institutes of Health (pending), Comments: 1) Imaging Genomics of the 22q11.two Deletion Syndrome; Radiological Society of North America Fellow Grant $(\$ 50,000), 2014-2015 ; 2)$ Integrated Neuroimaging Genomic Ontology Toolbox: a family of statistical computing algorithms for hypothesis-driven imaging genetic and longitudinal neuroimaging analysis; National Institutes of Health Mentored Career Development Award in Biomedical Big Data Science for Clinicians and Doctorally Prepared Scientists (K01, submitted); Payment for Lectures (including service on Speakers Bureaus): Registration waived for 3 invited lectures; no stipend or other compensation, Comments: 1) J.E. Schmitt, M.C. Neale, J.N. Giedd; "Genetically-Informative Multivariate Models of Brain Maturation," Imaging Genetics Symposium, Behavioral Genetics Association, San Diego, California (2015); 2) J.E. Schmitt; Outstanding Paper: The Dynamic Role of Genetics on Cortical Patterning During Childhood and Adolescence, American Society for Neuroradiology, Chicago, Illinois (2015); 3) T.S. Cook, J.E. Schmitt, J.C. Wildenberg: Introduction to Statistical Computing with R, Society for Imaging Informatics in Medicine, Washington DC (2015).

\section{REFERENCES}

1. Boon JM, Abrahams PH, Meiring JH, et al. Lumbar puncture: anatomical review of a clinical skill. Clin Anat 2004;17:544-53 CrossRef Medline

2. Stiffler KA, Jwayyed S, Wilber ST, et al. The use of ultrasound to identify pertinent landmarks for lumbar puncture. Am J Emerg Med 2007;25:331-34 CrossRef Medline

3. Edwards C, Leira EC, Gonzaelez-Alegre P. Residency training: a failed lumbar puncture is more about obesity than lack of ability. Neurology 2015;84:e69-72 CrossRef Medline

4. Williams MT. The oblique interlaminar approach for fluoroscopyguided lumbar puncture: keep the eye opened! Diagn Interv Imaging 2014;95:629-32 CrossRef Medline

5. Wang Y, Beydoun MA. The obesity epidemic in the United States: gender, age, socioeconomic, racial/ethnic, and geographic characteristics - a systematic review and meta-regression analysis. Epidemiol Rev 2007;29:6-28 CrossRef Medline

6. Halpenny D, O’Sullivan K, Burke JP, et al. Does obesity preclude lumbar puncture with a standard spinal needle? The use of computer tomography to measure the skin to lumbar subarachnoid space distance in the general hospital population. Eur Radiol 2013; 23:3191-96 CrossRef Medline

7. Yu SD, Chen MY, Johnson AJ. Factors associated with traumatic fluoroscopy-guided lumbar punctures: a retrospective review. AJNR Am J Neuroradiol 2009;30:512-15 CrossRef Medline

8. Boddu SR, Corey A, Peterson R, et al. Fluoroscopic-guided lumbar puncture: fluoroscopic time and implications of body mass index: a baseline study. AJNR Am J Neuroradiol 2014;35:1475-80 CrossRef Medline

9. The Joint Commission. Sentinel Event Alert. Issue 47: Radiation risks of diagnostic imaging. 2011. http://www.jointcommission.org/ sea_issue_47/. Accessed February 11, 2015

10. Abe KK, Yamamoto LG, Itoman EM, et al. Lumbar puncture needle length determination. Am J Emerg Med 2005;23:742-46 CrossRef Medline

11. Ma HP, Hung YF, Tsai SH, et al. Predictions of the length of lumbar puncture needles. Comput Math Methods Med 2014;2014:732694 CrossRef Medline

12. Stocker DM, Bonsu B. A rule based on body weight for predicting the optimum depth of spinal needle insertion for lumbar puncture in children. Acad Emerg Med 2005;5:105-06

13. Chong SY, Chong LA, Ariffin H. Accurate prediction of the needle depth required for successful lumbar puncture. Am J Emerg Med 2010;28:603-06 CrossRef Medline

14. Kieffer S, Mukundan S, Rollins N. ACR-ASNR-SPR Practice Guideline for the Performance of Myelography and Cisternography. 2013. http://www.acr.org/ /media/f4c49aa1834d46a081f5f0ff20e1e26b. pdf. Accessed February 12, 2015

15. Chatha DS, Schweitzer ME. MRI criteria of developmental lumbar spinal canal stenosis revisited. Bull NYU Hosp Jt Dis 2011;69:303-07 Medline

16. Saleem S, Aslam HM, Rehmani MAK, et al. Lumbar disc degenerative disease: disc degeneration symptoms and magnetic resonance image findings. Asian Spine J 2013;7:322-34 CrossRef Medline

17. National Heart, Lung, and Blood Institute. How Are Overweight and Obesity Diagnosed? http://www.nhlbi.nih.gov/health/health-topics/ topics/obe/diagnosis.html. Accessed August 6, 2015

18. Dixon AK. Abdominal fat assessed by computed tomography: sex difference in distribution. Clin Radiol 1983;34:189-91 CrossRef Medline

19. Toth MJ, Tchernof A, Sites CK, et al. Effect of menopausal status on body composition and abdominal fat distribution. Int J Obes Relat Metab Disord 2000;24:226-31 CrossRef Medline

20. Abel AS, Brace JR, McKinney AM, et al. Practice patterns and opening pressure measurements using fluoroscopically guided lumbar puncture. AJNR Am J Neuroradiol 2012;33:823-25 CrossRef Medline

21. Finucane MM, Stevens GA, Cowan MJ, et al; Global Burden of Metabolic Risk Factors of Chronic Diseases Collaborating Group (Body Mass Index). National, regional, and global trends in body-mass index since 1980: systematic analysis of health examination surveys and epidemiological studies with 960 country-years and $9 \cdot 1$ million participants. Lancet 2011;377:557-67 CrossRef Medline

22. Carnie J, Boden J, Gao Smith F. Prediction by computerised tomography of distance from skin to epidural space during thoracic epidural insertion. Anaesthesia 2002;57:701-04 CrossRef Medline 